\title{
A Feed Forward Artificial Neural Nework for the Stock Market Forecasting Using Conventional Forecasts as Input Variables
}

\author{
T.K.K.R. Mediliyegedara ${ }^{1}$, L.H.P. Gunaratne ${ }^{2}$, \\ ${ }^{1}$ School of Engineering, Science and Design, Glasgow Caledonian University, \\ Glasgow, U.K. \\ ${ }^{2}$ Department of Agricultural Economics and Business Management, University of \\ Peradeniya, Peradeniya, Sri Lanka
}

E-mail: K.Mediliyegedara@gcal.ac.uk

\begin{abstract}
In this study, first, Milanka Price Index (MPI) of the Colombo Stock Exchange (CSE) was forecast using Linear Moving average (LMA), Simple Exponential Smoothing (SES) and Adaptive Response Religh's Single exponential Smoothing (ARRSES). Then, a Feed - Forward Artificial Neural Network (FFANN) approach was developed where the inputs of the neural network are the forecasted values from conventional forecasting techniques. Mean Absolute Percentage Error (MAPE) and Prediction Error Variance (PEV) were employed to measure the performance of LMA, SES, ARRESES and the proposed FFANN method. Finally, the results of the conventional approaches have been compared with that of the proposed FFANN approach.
\end{abstract}

\section{Introduction}

Forecasting is clearly a necessary and important endeavor for business organizations and investors in planning for the future. Most forecasting techniques used today are based upon traditional linear or nonlinear statistical models, such as regression analysis or time series analysis. Although these models are useful and have been utilized for many years to predict, the models are somewhat limited in their ability to forecast in certain situations.

Among the novel approaches available, neural network approach to be of growing interest. Fletcher and Goss [1] compared the performance of neural networks with linear regression and determined that the neural network predict bankruptcy more accurately than the statistical techniques. Coats and Fant [2] compared the performance of neural networks with multiple discriminant analysis in firm failure prediction, and found that the neural networks perform better. On the other hand Altman et al. [3] found the performance of discriminant analysis and neural networks in firm failure prediction to be about the same. In the area of bank failures predictions Salchenberger et al. [4] found neural networks more accurate than logistic regression, while Tam and Kiang [5] compared neural networks with discriminant analysis and logistic regression, and found neural networks to perform better than all of these tech- niques.

Amir [6] developed an ANN bankruptcy prediction model and proposed novel indicators for the ANN system in the area of bankruptcy prediction. Further, Amir [6] shows that the use of these novel indicators in addition to traditional financial ratio indicators provide a significant improvement in the prediction. Skabar and Cloete [7] described a methodology by which neural networks could be trained indirectly, using a genetic algorithm based weight optimization procedure, to determine buy and sell points for financial commodities traded on a stock exchange. Wei-Wang and Frank [8] demonstrated an application of ANNs to predict future trends in Shanghai Stock Exchange (SSE) in China. They used two approaches to predict future trends in SSE such as Seasonal Autoregressive Integrated Moving Average (SARIMA) method and Feed Forward Neural Network (FFNN) method. They further argued that the Neural Network's topology greatly influence the results. This paper presents FFANN approach for stock market forecasting where the inputs of neural network are the forecast values from conventional forecasting techniques.

\section{Colombo Stock Exchange (CSE) [9]}

The Colombo Stock Exchange is the organization responsible for the operation of the Stock Market in Sri Lanka. The shares of all listed companies are traded on this market, which are freely accessible to any buyer or seller. In addition, a company's debentures and other type of securities can also be transacted here. The CSE was established under the Companies Act No. 17 of 1982 and licensed by the Securities and Exchange Commission of Sri Lanka (SEC) to operate as the Stock Exchange in Sri Lanka.

Securities and Exchange Commission of Sri Lanka (SEC) is a statutory body set up by the government to regulate and oversee the securities market. The objective of the SEC is the creation and maintenance of a fair and orderly securities market. It is responsible for overseeing the regulation of the market and ensuring that professional standards are maintained. Other duties of the SEC include 
protection of investors through the operation of a compensation fund to protect them from financial losses arising from a broker failing to meet his contractual obligations.

CSE is consisted of 241 listed companies at the end of 2003. Total market capitalization of CSE was SLR. 320 billions, which is $18 \%$ of Sri Lankan GDP.

\subsection{Price Indices in Colombo Stock Exchange (CSE)}

The CSE has two types of price indices such as Sector Price Indices and Main Price Indices. Main Price Indices are capital weighted indices. The weight of any company is taken as the number of ordinary shares listed on the market This weighting system allows the price movements of larger companies to have a greater impact on the index. Such a weighting system was adopted on the assumption that the general economic situation has a greater influence on larger companies than on smaller ones.

The daily trading activity results in market price changes and this in turn changes market capitalization, which affects price indices. There are, however, exceptions other than market price changes. Sometimes the number of listed shares will increase or decrease and this will affect the market capitalization of companies. In order to avoid the fluctuation of Price Indices, the Base Market Capitalization and Market Capitalization are adjusted in such instances.

There are two Main Price Indices such as All Share Price Index (ASPI) and Milanka Price Index (MPI). This study is focused on MPI. The MPI was introduced in January 1999. The MPI identifies the movement of share prices of blue-chip companies in CSE. The base index of the MPI is set at 1000 points as at 31st December 1998.

\subsection{Forecasting Milanka Price Index (MPI) with ANNs}

An accurate forecasting of MPI value is very useful in many business and governmental organizations to make various decisions. It helps investors to make investment decisions, helps policy makers to formulate policy and useful for donors to make decision regarding donations.

There are two criteria, which are taken into account in the construction of the Milanka Price Index. First is the size of the companies, which is measured by market capitalization. The second is the liquidity of the companies which is measured by the number of trades executed, and the trading value as a percentage of the average market capitalization. However, Elyasiani, Perera and Puri [10] argued that the CSE suffers from lack of market liquidity and from high concentration of market capitalization in blue chip stocks. Most local investors hold on to their stocks, especially blue chips, for long period of time without trading because of the perception that they will not be able to buy again later if they so desire. Therefore, one can argue that CSE has a unique behavior hence a unique dynamical behavior of the MPI.

\section{Limitations of Conventional Forecasting Techniques}

Over the last few decades, there has been much research directed at predicting the stock markets and making better decisions. Most of these techniques have been based on statistical techniques and judgmental methods. Statistical techniques include time series modeling and regression analysis. Some statistical time series methods have inherent limitations due to the way in which the models are estimated. Therefore, there are many weaknesses in such conventional forecasting techniques. Regression forecasting models are based on the assumption that the data set is normally distributed. This is no longer a valid assumption for the stock market data. Judgmental methods demands experts and lack of credibility. Therefore, it is crucial to investigate more sophisticated forecasting techniques such as ANNs to detect complex relationships while allowing for high levels of noisy data and chaotic components for Colombo Stock Exchange.

\section{Strengths of Artificial Neural Network (ANN) Approach for the Forecasting}

Neural networks, with their remarkable ability to drive meaning fully from complicated or imprecise data, can be used to extract patterns and detect trends that are too complex to be noticed by either humans or other computer techniques. A trained neural network can be thought of as an "expert" in the category of information it has been given to analyze. This expert can then be used to provide projections given new situations of interest and answer "what if" questions.

Other features include adaptive learning, self-organization, real time operation and fault tolerance. Adaptive learning is the ability to learn how to do tasks based on the data given for training or initial experience. Self-organization means the ability to create its own organization or representation of the information it receives during the learning time. It is possible to carry out computations of an ANN in parallel, which provides the basis for the real time operation. Partial distortion of a network leads to the corresponding degradation of performance. Due to the ability of fault tolerance, some network capabilities may be retained even with major network damages.

The literature suggests that several potential advantages that ANNs have over statistical methods. ANNs can deal with very complex and highly non-linear data. ANNs make no assumptions about the nature of the data set. It has ability of identifying non-linear relationships in the data set. ANNs not only can estimate non-linear functions but also they can extract any residual non-linear elements of the data after linear terms are removed. With ANNs using one or more hidden layers, the 
networks can partition the sample space automatically and build different functions in different portions of the sample space. This means that ANNs have a capability of building piece-wise non-linear models [11]. On the other hand, ANNs model can deal with missing or incomplete data. The estimation of many kinds of statistical time series models requires human interaction and evaluation. However, the estimation of artificial neural networks can be automated [12]. Many statistical models must be re-estimated periodically when new data arrive whereas many ANNs can be trained incrementally.

\section{Data Set and Pre-processing}

Milanka Price Index (MPI) values were obtained from Colombo Stock Exchange (CSE) for the period of 175 weeks form 04/01/1999. Since CSE does not operate in weekends and holidays, weekly averaged MPI values were calculated using equation 1 . Weekly averaged MPI can be defined as,

$M P I W K=\frac{\sum_{i}^{p} M P I_{i}}{p}$

Where,

$p \quad$ - Total number of days per week in which CSE operates,

MPI - Daily Milanka Price Index.

\section{Methodology}

Three conventional techniques were used to predict weekly averaged MPI such as Linear Moving average (LMA), Simple Exponential Smoothing (SES) and Adaptive Response Religh's Single exponential Smoothing (ARRSES). Then a neural network approach was used. The effectiveness of estimated values was measured by Mean Absolute Percentage Error (MAPE) and Prediction Error Variance (PEV).

\subsection{Linear Moving average (LMA)}

The LMA forecasting procedure involves three aspects [13]. Firstly, a single moving average at time $t$, which is denoted by $S_{t}^{\prime}$, is incorporated. Secondly, an adjustment, which is the difference between the single and the double moving average at time $t$ (denoted by $S_{t}^{\prime}-S_{t}^{\prime \prime}$ ), has to be incorporated. Then, an adjustment for trend from period $t$ to $(t+m)$ is incorporated where $m$ is the number of periods ahead to be forecast. Linear moving average can be defined as follows:

$F_{t+m}=a_{t}+b_{t} \cdot m$

Where,

$a_{t}=S_{t}^{\prime}+\left(S_{t}^{\prime}-S_{t}^{\prime \prime}\right)=2 S_{t}^{\prime}-S_{t}^{\prime \prime}$
$b_{t}=\frac{2}{N-1}\left(S_{t}^{\prime}-S_{t}^{\prime \prime}\right)$

$S_{t}^{\prime \prime}=\frac{S_{t}^{\prime}+S_{t-1}^{\prime}+S_{t-2}^{\prime}+\ldots+S_{t-N+1}^{\prime}}{N}$

$S_{t}^{\prime}=\frac{X_{t}+X_{t-1}+X_{t-2}+\ldots+X_{t-N+1}}{N}$

$\mathrm{N} \quad$ - Number of period used to calculate moving average

$S_{t}^{\prime} \quad$ - Single moving average for time $t$

$S_{t}^{/ /} \quad$ - Double moving average for time $t$

$X_{t} \quad$ - Actual value at time $t$

\subsection{Single Exponential Smoothing (SES)}

Exponential smoothing methods use an exponentially decreasing weights as the observation get older. Single Exponential Smoothing (SES) can be defined as follows.

$F_{t+1}=F_{t}+\alpha\left(A_{t}+F_{t}\right)$

Where,

$F_{t+1} \quad$ - Forecast value for time $t+1$

$F_{t} \quad$ - Forecast value for time $t$

$A_{t} \quad$ - Actual value at time t

$\alpha \quad$ - Smoothing constant $(0 \leq \alpha \leq 1)$

When $\alpha$ is close to one, the new forecast will include a substantial adjustment for the error in the previous forecast. Conversely, when $\alpha$ is close to zero, the new forecast will include very little adjustment. Thus, the effect of a large and small $\alpha$ is completely analogous to the effect of including a small or a large number of observations when computing a moving average. SES requires little storage and few computations.

\subsection{Adaptive Response Religh's Single Exponential Smoothing (ARRSES)}

Adaptive Response Religh's Single Exponential Smoothing (ARRSES) method allows $\alpha$ value to change in a controlled manner according to changes in the pattern of data. The following equations can be used to calculate forecast values.

$F_{t+1}=\alpha_{t} X_{t}+\left(1-\alpha_{t}\right) F_{t}$

Where,

$\alpha_{t+1}=\left|\frac{E_{t}}{M_{t}}\right|$

$E_{t}=\beta \cdot e_{t}+(1-\beta) \cdot E_{t-1}$ 
$M_{t}=\beta\left|e_{t}\right|+(1-\beta) M_{t-1}$

$e_{t}=X_{t}-F_{t}$

$E_{t} \quad$ - Smoothed error term

$M_{t} \quad$ - Smoothed absolute error term

$\alpha_{t}, \beta_{t}$ - Smoothing constants $\left(0 \leq \alpha_{t} \leq 1,0 \leq \beta_{t} \leq 1\right)$

\subsection{Forecasting with a ANN Model}

Architecture of the ANN Model, which is shown in Figure 1, is a Feed Forward Artificial Neural Network (FFANN). There are nine neurons in the input layer. FFANN consists of one hidden layer. Output layer consists of one neuron. Logistic Sigmoid Function (LOGSIG) was used as the activation function throughout the neural network. $\mathrm{A}(\mathrm{t})$ and $\mathrm{F}(\mathrm{t}+1)$ are the actual value at time $\mathrm{t}$ and the forecast value at time $t+1$ respectively. $I_{1}(t+1), I_{2}(t+1)$ and $I_{3}(t+1)$ are the forecasted values for time $t+1$ using LMA, SES and ARRSES techniques respectively.

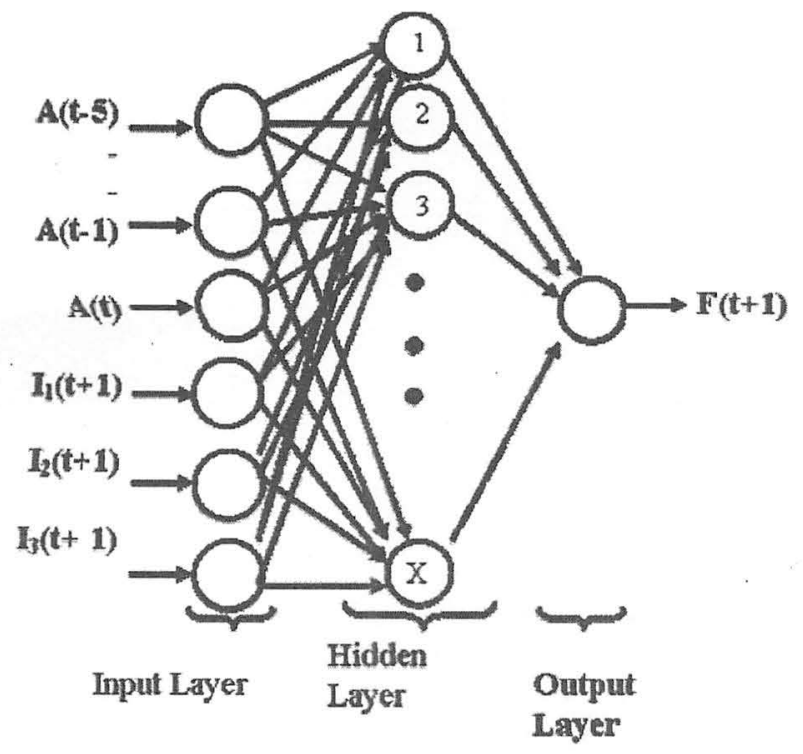

Figure 1: Architecture of the ANN Model

\subsection{Performance Measures}

It is crucial to measure the performance of a forecasting technique to ensure the accuracy of forecast values. Virtanen and Yli-Olli [14] employed Mean Error (ME), Mean Absolute Error (MAE), Mean of Squired Error (MSE), Root Mean of Squired Error (RMSE), Mean Percentage Error (MPE) and Mean Absolute Percentage Error (MAPE) when comparing univariate time series with multivariate econometric models for stock market price forecasting in Helsinki Stock Exchange in Finland.

In this study, the effectiveness of estimated values was measured by evaluating the prediction accuracy, which was calculated using Mean Absolute Percentage Error
(MAPE) and Prediction Error Variance (PEV). The Mean Absolute Percentage Error (MAPE), as the name suggests, is the mean or average value of the absolute forecasting errors as a percentage of the respective data value. MAPE can be defined as:

$$
M A P E=\left\{\frac{1}{n} \times \sum_{t=0}^{n-1} \frac{\left|y_{t}-\hat{y}_{t}\right|}{y_{t}}\right\} x 100 \%
$$

Where, $y_{t}$ is the actual value at time t. $\hat{y}_{t}$ is the predicted value for time $t$. " $n$ " is the number of data points. The smaller the value of MAPE, the better the prediction model.

Prediction error variance is defined as:

$$
\sigma_{y}^{2}=E\left\{\left(y_{t}-\hat{y}_{t}\right)^{2}\right\}
$$

Where $\sigma_{y}^{2}$ is the prediction error variance, $y_{t}$ is the actual value at time $\mathrm{t} ; \hat{y}_{t}$ is the predicted value for time $\mathrm{t} ; E\{\}$ is the expectation operator. The smaller the value of prediction error variance, the better the prediction model.

\section{Results}

MAPE for LMA, SES, ARRSES and proposed FFANN model are $5.00 \%, 4.01 \%, 3.55 \%$ and $0.91 \%$ respectively. On the other hand, MAPE for LMA, SES, ARRSES and FFANN model are 3959.05, 2890.62, 2366.25 and 975 respectively. It is clear from MAPE and PEV values that the properly trained FFANNs are outperform the other conventional forecasting techniques like LMA, SES and ARRSES.

\section{Conclusions}

Forecasting techniques such as LMA, SES, ARRSES and FFANN have been compared using PEV and MAPE. In this FFANN the inputs were the forecasts developed by LMA, SES and ARRSES. The experimental results show that the properly trained FFANNs are outperformed the other conventional forecasting techniques such as LMA, SES and ARRSES. Further more, it also demonstrate that FFANNs are good at predicting stock market data because of its ability of incorporating synergy of various forecasting techniques. This ensures that proposed ANN model is suitable for actual forecasting in Colombo Stock Exchange.

\section{References}

[1] Fletcher, D. and Goss, E., Forecasting with neural networks: An application using bankruptcy data, Informa- 
tion and Management, Vol. 24, No. 3, pp. 159-167, 1993.

[2] Coats, P.K. and Fant, L.F., A neural network approach to forecasting financial distress, Journal of Business Forecasting, Vol. 10, No. 4, pp. 9-12, 1991.

[3] Altman, E.I., Marco, G., and Varetto, F., Corporate distress diagnosis: Comparisons using linear discriminant analysis and neural networks (the Italian experience), Journal of Banking and Finance, Vol. 18, No. 3, pp. 505-529, 1994.

[4] Salchenberger, L. M., Cinar, E. M., and Lash, N. A., Neural networks: A new tool for predicting thrift failures, Decision Sciences, Vol. 23, No. 4, pp. 899-916, 1992.

[5] Tam, K. Y. and Kiang, M. Y., Managerial applications of neural networks: The case of bank failure predictions, Management Science, Vol. 38, No. 7, pp. 926-947, 1992.

[6] Amir, F. A., Bankruptcy Prediction for Credit Risk Using Neural Networks: A Survey and New Results, IEEE Transactions on Neural Networks, Vol. 12, No. 4, pp. 929-935, 2001.

[7] Skabar A. and Cloete A., Neural Networks, Financial Trading and the Efficient Markets Hypothesis, 9th International Conference on Neural Information Processing, ISBN: 981-04-7525-X, 2002.

[8] Wei-Wang, D.O and Frank C. L., Future Trend of Shanghai Stock Market, 9th International Conference on Neural Information Processing, ISBN: 981-04-7525-X, 2002.

[9] Annual Report, Colombo Stock Exchange, Colombo, Sri Lanka, 2003.

[10] Elyasiani, E., Perera, P. and Puri, N., Interdependence and dynamic linkages between stock markets of Sri Lanka and its trading partners, Journal of Multinational Financial Management, Vol. 8, pp. 89-101, 1998.

[11] Wasserman, P.D., Neural Computing: Theory and Practice, Van Nostrand Reinhold: New York, pp. 30-33, 1989.

[12] Hoptroff, R.G., The principles and practice of Time Series Forecasting and Business Modeling Using Neural Nets, Neural Computing and Applications, Vol.1, pp. 59-66, 1993.

[13] Makridakis, S., Wheelwright, S. and McGee, V., Forecasting Methods and Applications, Second Edition, John Wiley \& Sons, New York, ISSN 0271-6046, 1983.

[14] Vittanen, I. and Yli-Olli, P., Forecasting Stock Market Price in a Thin Security Market, Omega: International Journal of Management Science, Vol. 15, No.2, pp. 145-155, 1987. 